\title{
A measure of the size of the magnetospheric accretion region in TW Hydrae
}

https://doi.org/10.1038/s41586-020-2613-1

Received: 25 January 2020

Accepted: 8 June 2020

Published online: 26 August 2020

Check for updates

\section{GRAVITY Collaboration*}

Stars form by accreting material from their surrounding disks. There is a consensus that matter flowing through the disk is channelled onto the stellar surface by the stellar magnetic field. This is thought to be strong enough to truncate the disk close to the corotation radius, at which the disk rotates at the same rate as the star. Spectro-interferometric studies in young stellar objects show that hydrogen emission (a well known tracer of accretion activity) mostly comes from a region a few milliarcseconds across, usually located within the dust sublimation radius ${ }^{1-3}$. The origin of the hydrogen emission could be the stellar magnetosphere, a rotating wind or a disk. In the case of intermediate-mass Herbig AeBe stars, the fact that Brackett $\gamma$ $(\mathrm{Br} \gamma)$ emission is spatially resolved rules out the possibility that most of the emission comes from the magnetosphere ${ }^{4-6}$ because the weak magnetic fields (some tenths of a gauss) detected in these sources ${ }^{7,8}$ result in very compact magnetospheres. In the case of T Tauri sources, their larger magnetospheres should make them easier to resolve. The small angular size of the magnetosphere (a few tenths of a milliarcsecond), however, along with the presence of winds ${ }^{9,10}$ make the interpretation of the observations challenging. Here we report optical long-baseline interferometric observations that spatially resolve the inner disk of the T Tauri star TW Hydrae. We find that the near-infrared hydrogen emission comes from a region approximately 3.5 stellar radii across. This region is within the continuum dusty disk emitting region (7 stellar radii across) and also within the corotation radius, which is twice as big. This indicates that the hydrogen emission originates in the accretion columns (funnel flows of matter accreting onto the star), as expected in magnetospheric accretion models, rather than in a wind emitted at much larger distance (more than one astronomical unit).
The T Tauri star TW Hydrae (TW Hya) belongs to an association of young stars around 8 million years old. Its proximity to Earth, as well as its favourable pole-on orientation ${ }^{11}$, makes it an ideal candidate for protoplanetary disk studies. The disk structure of TW Hya includes a dust-depleted inner hole, as well as a series of bright rings, with the closest one located at about one astronomical unit (1 AU) from the star ${ }^{11,12}$. The presence of the inner hole and the small near-infrared excess ${ }^{13,14}$ make TW Hya the prototypical 'transitional disk', in which planets and photoevaporation are expected to be the main mechanisms of disk dispersal. However, the measurement of non-negligible accretion rates $\left(2.3 \times 10^{-9} M_{\odot} \mathrm{yr}^{-1} \text {; where } M_{\odot} \text { is the solar mass }\right)^{15}$ indicates that the inner-disk region of TW Hya is still rich in gas. Further evidence of accretion is given by the detection of a nearly pole-on cool photospheric spot (stable over several years), coincident with the location of the main magnetic pole $(B \approx 2.5 \mathrm{kG})$, and a region of accretion-powered excess line emission ${ }^{16}$. This suggests that accretion in TW Hya takes place mostly poleward, and that the stellar magnetic field is strong enough to magnetically truncate the inner disk at a few stellar radii from the star. This value is equivalent to a few tenths of a milliarcsecond, and so it is impossible to directly resolve the magnetospheric accretion region, even for such a nearby star, using conventional methods. This leaves spectro-interferometry as the only suitable technique.

With this aim, we conducted high-angular resolution observations of the hydrogen Bry line in TW Hya using the Very Large Telescope Interferometer (VLTI) instrument GRAVITY with the four 8-m Unit Telescopes (Fig. 1). The Bry line is a well known tracer of accretion in low-mass protostars through an empirical relationship that relates the line and accretion luminosities ${ }^{17,18}$. Our interferometric measurements allowed us to probe the Bry line and $\mathrm{K}$-band emitting regions along six different baselines (projected baselines ranging from approximately $130 \mathrm{~m}$ to $45 \mathrm{~m}$, resulting in nominal angular resolutions of around 4 mas to 10 mas) and at various position angles. By fitting a geometrical model (see Methods) to the continuum emission (star plus continuum circumstellar emission) and assuming a K-band to stellar flux ratio of 1.18 (refs. $^{14,15}$ ), we derive a stellar radius of $R_{*}=(1.29 \pm 0.19) R_{\odot}$ (consistent with theoretical expectations; where $R_{\odot}$ is the solar radius and $R_{*}$ is the stellar radius) and a radius for the K-band continuum excess/ circumstellar emission of $R_{\text {circ }}=(6.50 \pm 0.16) R_{*}$ (see Table 1 and Fig. 2$)$. These values are in agreement with previous interferometric results and spectroscopic studies ${ }^{14,15,19}$. Furthermore, the location of the K-band 


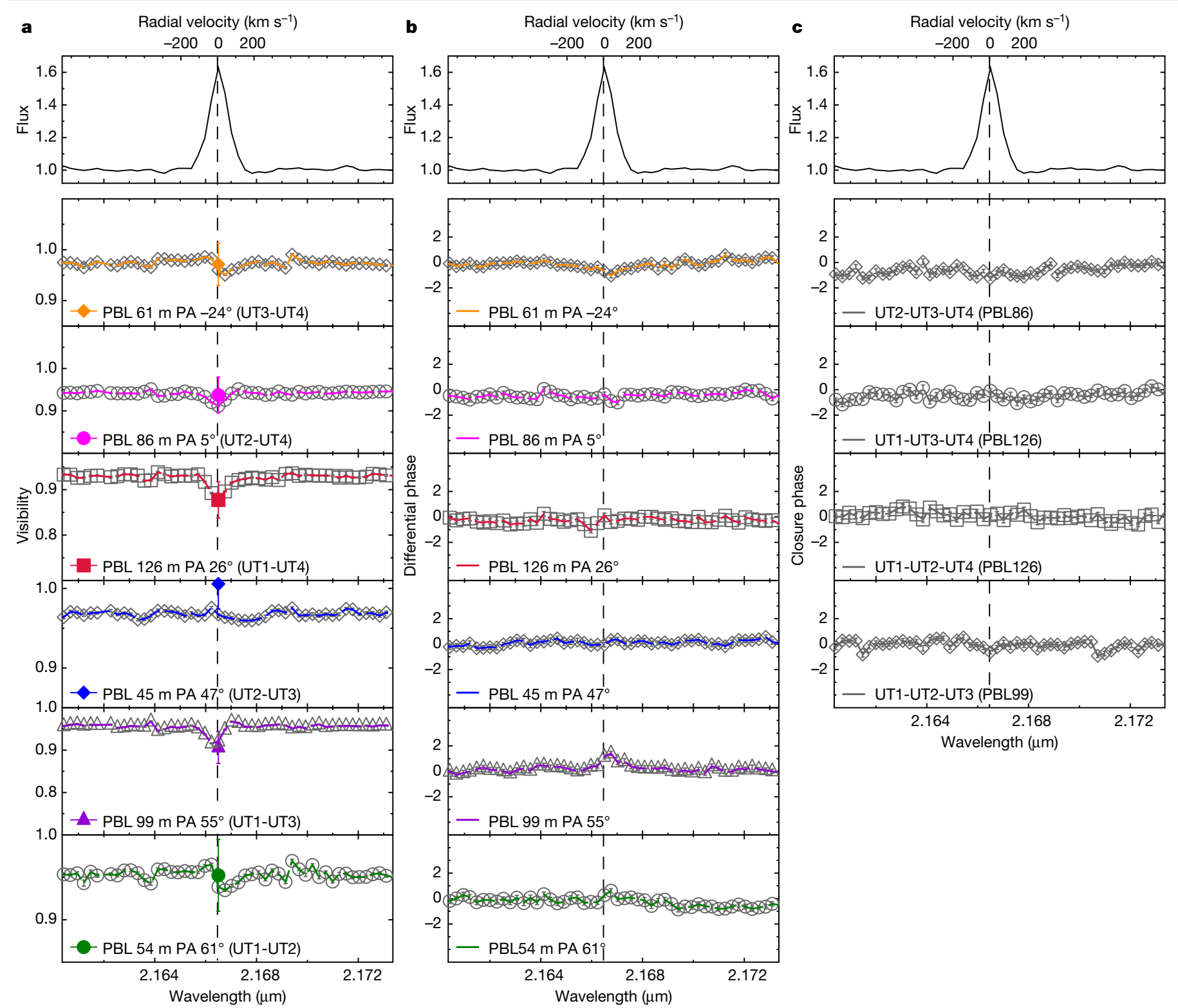

Fig. 1 | VLTI-GRAVITY observations of TW Hya. The Bry line profile is normalized to the continuum flux and shown in the top row. The radial velocity is with respect to the stellar reference. a, Wavelength-dispersed visibility amplitudes for the six baselines along with the errors derived from the data reduction. PBL, projected baseline; PA, position angle. The continuum-subtracted Bry line visibilities are shown as full coloured symbols. The associated errors were derived by

excess emission is consistent with the location of a disk rim, owing to silicate sublimation (see Methods).

By removing the continuum contribution to the line emission (see Methods), we find that the Bry line emitting region is very compact, but nonetheless marginally resolved for the longest projected baselines (more than about $60 \mathrm{~m}$ ). This allows us to measure a radius for the Bry emitting region of $R_{\mathrm{Bry}}=(3.49 \pm 0.20) R_{*}$ assuming a distance of about $60 \mathrm{pc}$ from the Sun to TW Hya (see Table 1, Fig. 2 and Extended Data Fig. 1). This size is consistent with the small (less than about $1^{\circ}$; with total amplitude of less than $2^{\circ}$ ) photocentre shift of the line with respect to the continuum (the so-called differential phase) detected in our longest baselines (see Fig. 1). Such a differential phase roughly translates into a $\mathrm{Br}$ line displacement of less than about $5 R_{*}$ (see Methods for more details), in agreement with the value derived from the continuum-subtracted Bry line visibilities. propagating equation (1) as described in the main text. $\mathbf{b}$, Same as a, but for the wavelength-dispersed differential-phase signals. Errors are derived from the data reduction pipeline. $c$, Wavelength-dispersed closure phases for the triplets UT2-UT3-UT4, UT1-UT3-UT4, UT1-UT2-UT4 and UT1-UT2-UT3. The maximum projected baseline of each baseline is indicated in parentheses. Errors are derived from the data reduction pipeline.

The inferred size of the Bry line emission is too compact to be emitted in a photoevaporative wind that in TW Hya is expected to be launched beyond the dust cavity $\left(R>0.5-1 \mathrm{AU} \text {, that is, } R>80 R *-160 R_{*}\right)^{20,21}$. It should be pointed out that in TW Hya there is no evidence of the presence of a disk wind, which is typically emitted within $0.5 \mathrm{AU}$ from the

\section{Table 1 | Size estimates of TW Hya}

\begin{tabular}{llll}
\hline TW Hya & $R$ (mas) & $R(\mathrm{AU})$ & $R\left(\times R_{\odot}\right)$ \\
\hline Star & $0.10 \pm 0.01$ & $0.006 \pm 0.001$ & $1.29 \pm 0.19$ \\
\hline Disk & $0.65 \pm 0.02$ & $0.039 \pm 0.001$ & $8.39 \pm 0.21$ \\
\hline Line & $0.35 \pm 0.02$ & $0.021 \pm 0.001$ & $4.50 \pm 0.26$
\end{tabular}

Estimates are derived from the best fit of the continuum and continuum-compensated Bry line data. 


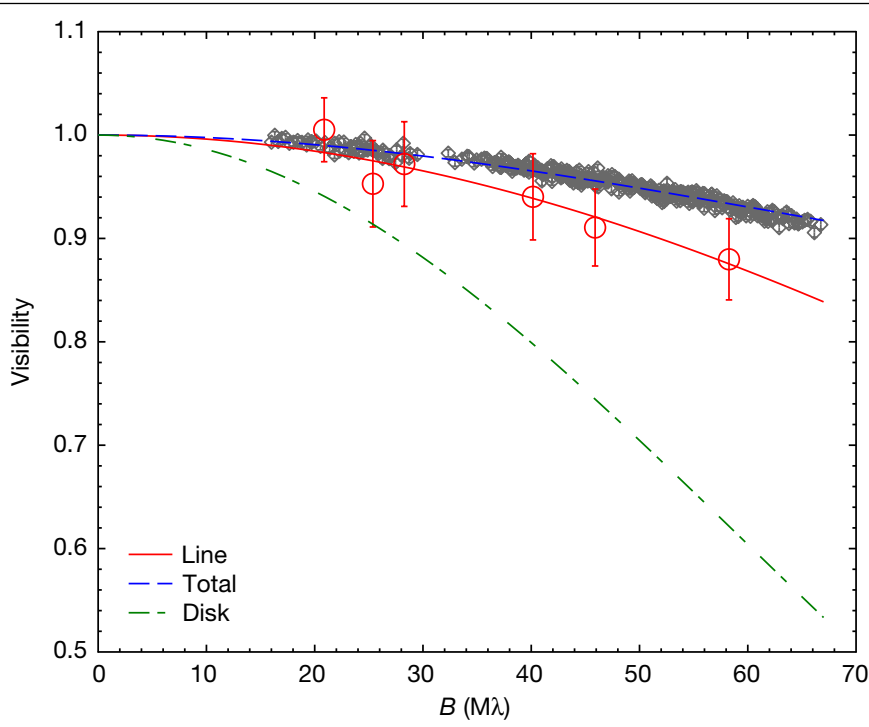

Fig. 2 | Visibility plot of TW Hya. Visibility points versus megalambda for the continuum calibrated fringe tracker data (open grey diamonds) and the continuum-subtracted $\mathrm{Br}$ line (open red circles). Spatial frequency is given in units of megalambda: $(\mathrm{PBL}(\mathrm{m}) /$ wavelength $(\mu \mathrm{m})$. The fit to the total continuum and continuum-subtracted Bry visibilities is shown in dashed blue and solid red lines. The circumstellar/disk visibility curve is also shown in dashed-dotted green line as reference. Error bars are derived from standard error propagation, as described in Methods.

source, or a jet, which would be associated with bright fast blue-shifted emission in lines, such as $\mathrm{H} \alpha$ and [O I] 6,300 $\AA$ and [S II] 6,717 $\AA$, that are not observed in this object ${ }^{9,22}$. Therefore, the results presented here indicate that the $\mathrm{Br} \gamma$ line is emitted in the magnetospheric accretion region. Classical magnetospheric accretion models assuming free-fall velocities along an axisymmetric, dipolar magnetosphere predict indeed that the Bry line is formed along the accretion columns ${ }^{23-25}$. In these models, the Bry line has a broad profile, comparable to the free-fall velocity, centred around zero velocity. This is the case for the Bry line observed in TW Hya, which shows a full width at zero intensity of about $400 \mathrm{~km} \mathrm{~s}^{-1}$, consistent with the expected velocity of gas around a solar-mass star falling at free-fall from about $3 R_{*}-4 R_{*}$. Therefore, our measurements indicate that the Bry line is emitted along the magnetospheric accretion columns that truncate the disk at around $3.5 R *$.

Is this value consistent with the expected magnetospheric truncation radius of TW Hya as determined by its magnetic field? Zeeman-Doppler imaging has been used to reconstruct the magnetic field topology and strength in TW Hya ${ }^{16}$. Those measurements showed that the magnetic field of TW Hya is strong (about $1.5 \mathrm{kG}$ ) and mostly poloidal and axisymmetric with respect to the stellar rotation axis. The field can be separated into a complex octupole component of approximately $2.5 \mathrm{kG}$ and a much fainter dipolar large-scale field of 400-700 G. Models for such complex magnetic field topologies show that the gas initially accretes following the dipolar field lines, although near the stellar surface the octupole component alters the flow of matter ${ }^{26,27}$. In accordance with this idea, and with the theoretical work of Bessolaz et al. ${ }^{28}$, we estimate a truncation radius of $3 R_{*}-4 R_{*}$ assuming a stellar radius and mass of $1.22 R_{\odot}$ and $0.6 M_{\odot}\left(\right.$ ref. $\left.^{15}\right)$, and a mass accretion rate of $2.3 \times 10^{-9} M_{\odot} \mathrm{yr}^{-1}$ (refs. ${ }^{14,15}$ ) for TW Hya, and a strength of the dipolar magnetic field component of 400-700 G. Therefore, the size of the Bry line emitting region derived from our interferometric measurements and the size of the truncation radius estimated from the magnetic field of TW Hya are strikingly similar. In addition, the measured size of the line-emitting region is inconsistent with a disk wind, since it is much smaller than the inferred truncation radius. There is a small possibility that dust-free disk gas extends inwards of the inferred sublimation radius, and could be responsible for at least part of the line emission. However, the previously measured magnetic field strength and geometry implies a disk truncation radius consistent with the size of the K-band continuum. Finally, the detection of spatially resolved line emission rules out the possibility that most of the Bry emission originates at the accretion shock near the stellar surface. A schematic view of our findings is shown in Extended Data Fig. 2. Our results are thus in agreement with the topology and strength of the magnetic field and they validate the assumption that when the magnetic field of the central star is complex, the truncation radius is located closer to the central star than would be expected if the magnetic field has a dipolar morphology of similar average strength ${ }^{26,29}$.

\section{Online content}

Any methods, additional references, Nature Research reporting summaries, source data, extended data, supplementary information, acknowledgements, peer review information; details of author contributions and competing interests; and statements of data and code availability are available at https://doi.org/10.1038/s41586-020-2613-1.

1. Kraus, S. et al. The origin of hydrogen line emission for five Herbig Ae/Be stars spatially resolved by VLTI/AMBER spectro-interferometry. Astron. Astrophys. 489, 1157-1173 (2008).

2. Eisner, J. A. et al. Near-infrared interferometric, spectroscopic, and photometric monitoring of T Tauri inner disks. Astrophys. J. 669, 1072-1084 (2007).

3. Perraut, K. et al. A disk wind in $\mathrm{AB}$ Aurigae traced with $\mathrm{Ha}$ interferometry. Astron. Astrophys. 596, A17 (2016).

4. Kurosawa, R. et al. Probing the wind-launching regions of the Herbig Be star HD 58647 with high spectral resolution interferometry. Mon. Not. R. Astron. Soc. 457, 2236-2251 (2016).

5. Garcia Lopez, R. et al. Probing the accretion-ejection connection with VLTI/AMBER. High spectral resolution observations of the Herbig Ae star HD 163296. Astron. Astrophys. 576, A84 (2015).

6. Caratti o Garatti, A. et al. AMBER/VLTI high spectral resolution observations of the Bry emitting region in HD 98922. A compact disc wind launched from the inner disc region. Astron. Astrophys. 582, A44 (2015)

7. Hubrig, S., Carroll, T. A., Schöller, M. \& Ilyin, I. The prevalence of weak magnetic fields in Herbig Ae stars: the case of PDS 2. Mon. Not. R. Astron. Soc. 449, L118-L122 (2015).

8. Alecian, E. et al. A high-resolution spectropolarimetric survey of Herbig $\mathrm{Ae} / \mathrm{Be}$ stars-I. Observations and measurements. Mon. Not. R. Astron. Soc. 429, 1001-1026 (2013).

9. Banzatti, A. et al. Kinematic links and the coevolution of MHD winds, jets, and inner disks from a high-resolution optical [O I] survey. Astrophys. J. 870, 76 (2019).

10. Simon, M. N. et al. Tracing slow winds from T Tauri stars via low-velocity forbidden line emission. Astrophys. J. 831, 169 (2016).

11. Huang, J. et al. $\mathrm{CO}$ and dust properties in the TW Hya disk from high-resolution ALMA observations. Astrophys. J. 852, 122 (2018)

12. van Boekel, R. et al. Three radial gaps in the disk of TW Hydrae imaged with SPHERE. Astrophys. J. 837, 132 (2017).

13. Calvet, N. et al. in Protostars and Planets IV 377-399 (Univ. Arizona Press, 2000).

14. Manara, C. F. et al. Gas content of transitional disks: a VLT/X-Shooter study of accretion and winds. Astron. Astrophys. 568, A18 (2014).

15. Venuti, L. et al. X-shooter spectroscopy of young stars with disks. The TW Hydrae association as a probe of the final stages of disk accretion. Astron. Astrophys. 632, A46 (2019).

16. Donati, J. F. et al. The large-scale magnetic field and poleward mass accretion of the classical T Tauri star TW Hya. Mon. Not. R. Astron. Soc. 417, 472-487 (2011).

17. Muzerolle, J., Hartmann, L. \& Calvet, N. A Bry probe of disk accretion in T Tauri stars and embedded young stellar objects. Astron. J. 116, 2965-2974 (1998).

18. Alcalá, J. M. et al. X-shooter spectroscopy of young stellar objects. IV. Accretion in low-mass stars and substellar objects in Lupus. Astron. Astrophys. 561, A2 (2014).

19. Akeson, R. L. et al. Radial structure in the TW Hya circumstellar disk. Astrophys. J. 728, 96 (2011).

20. Pascucci, I. et al. The photoevaporative wind from the disk of TW Hya. Astrophys. J. 736, 13 (2011).

21. Ercolano, B., Rosotti, G. P., Picogna, G. \& Testi, L. A photoevaporative gap in the closest planet-forming disc. Mon. Not. R. Astron. Soc. 464, L95-L99 (2017).

22. Fang, M. et al. A new look at T Tauri star forbidden lines: MHD-driven winds from the inner disk. Astrophys. J. 868, 28 (2018).

23. Muzerolle, J., Calvet, N. \& Hartmann, L. Emission-line diagnostics of T Tauri magnetospheric accretion. II. Improved model tests and insights into accretion physics. Astrophys. J. 550, 944-961 (2001).

24. Kurosawa, R., Romanova, M. M. \& Harries, T. J. Multidimensional models of hydrogen and helium emission line profiles for classical T Tauri stars: method, tests and examples. Mon. Not. R. Astron. Soc. 416, 2623-2639 (2011).

25. Gullbring, E., Hartmann, L., Briceno, C. \& Calvet, N. Disk accretion rates for T Tauri stars. Astrophys. J. 492, 323 (1998). 


\section{Article}

26. Gregory, S. G., Matt, S. P., Donati, J. F. \& Jardine, M. The non-dipolar magnetic fields of accreting T Tauri stars. Mon. Not. R. Astron. Soc. 389, 1839-1850 (2008).

27. Romanova, M. M., Long, M., Lamb, F. K., Kulkarni, A. K. \& Donati, J. F. Global 3D simulations of disc accretion on to the classical T Tauri star V2129 Oph. Mon. Not. R. Astron. Soc. 411, 915-928 (2011).

28. Bessolaz, N., Zanni, C., Ferreira, J., Keppens, R. \& Bouvier, J. Accretion funnels onto weakly magnetized young stars. Astron. Astrophys. 478, 155-162 (2008).

29. Johnstone, C. P. et al. Classical T Tauri stars: magnetic fields, coronae and star-disc interactions. Mon. Not. R. Astron. Soc. 437, 3202-3220 (2014).

Publisher's note Springer Nature remains neutral with regard to jurisdictional claims in published maps and institutional affiliations.

(C) The Author(s), under exclusive licence to Springer Nature Limited 2020

\section{GRAVITY Collaboration}

R. Garcia Lopez ${ }^{1,2,3}{ }^{冈}$, A. Natta ${ }^{2}$, A. Caratti o Garatti ${ }^{1,2,3}$, T. P. Ray ${ }^{2}$, R. Fedriani ${ }^{2,4}$, M. Koutoulaki ${ }^{2,5}$, L. Klarmann ${ }^{3}$, K. Perraut ${ }^{6}$, J. Sanchez-Bermudez ${ }^{3,7}$, M. Benisty ${ }^{6,8}$ C. Dougados $^{6}$, L. Labadie ${ }^{9}$, W. Brandner ${ }^{3}$, P. J. V. Garcia ${ }^{10,11,12}$, Th. Henning ${ }^{3}$, P. Caselli ${ }^{13}$ G. Duvert ${ }^{6}$, T. de Zeeuw ${ }^{13,14}$, R. Grellmann ${ }^{9}$, R. Abuter ${ }^{5}$, A. Amorim $^{11,15}$, M. Bauböck ${ }^{13}$, J. P. Berger ${ }^{5,6}$, H. Bonnet ${ }^{5}$, A. Buron ${ }^{13}$, Y. Clénet ${ }^{16}$, V. Coudé du Foresto ${ }^{16}$, W. de Wit $^{12}$, A. Eckart ${ }^{9,17}$, F. Eisenhauer $^{13}$, M. Filho ${ }^{10,11,12}$, F. Gao $^{13}$, C. E. Garcia Dabo ${ }^{5}$, E. Gendron ${ }^{16}$
R. Genzel ${ }^{13,18}$, S. Gillessen ${ }^{13}$, M. Habibi ${ }^{13}$, X. Haubois ${ }^{12}$, F. Haussmann ${ }^{13}$, S. Hippler ${ }^{3}$, Z. Hubert ${ }^{6}$, M. Horrobin ${ }^{9}$, A. Jimenez Rosales ${ }^{13}$, L. Jocou ${ }^{6}$, P. Kervella ${ }^{16}$, J. Kolb ${ }^{12}$, S. Lacour ${ }^{16}$,

J.-B. Le Bouquin ${ }^{6}$, P. Léna ${ }^{16}$, T. Ott ${ }^{13}$, T. Paumard ${ }^{16}$, G. Perrin ${ }^{16}$, O. Pfuhl ${ }^{5}$, A. Ramirez ${ }^{5}$, C. Rau ${ }^{13}$, G. Rousset ${ }^{16}$, S. Scheithauer ${ }^{3}$, J. Shangguan ${ }^{13}$, J. Stadler ${ }^{13}$, O. Straub $^{13}$, C. Straubmeier $^{9}$,

E. Sturm ${ }^{13}$, E. van Dishoeck ${ }^{13,14}$, F. Vincent ${ }^{16}$, S. von Fellenberg ${ }^{13}$, F. Widmann ${ }^{13}$, E. Wieprecht ${ }^{13}$, M. Wiest ${ }^{9}$, E. Wiezorrek ${ }^{13}$, J. Woillez ${ }^{5}$, S. Yazici ${ }^{9,13}$ \& G. Zins ${ }^{12}$

School of Physics, University College Dublin, Dublin, Ireland. ${ }^{2}$ Dublin Institute for Advanced Studies, Dublin, Ireland. ${ }^{3}$ Max Planck Institute for Astronomy, Heidelberg, Germany. ${ }^{4}$ Department of Space, Earth and Environment, Chalmers University of Technology, Gothenburg, Sweden. ${ }^{5}$ European Southern Observatory, Garching, Germany. ${ }^{6}$ Université Grenoble Alpes, CNRS, IPAG, Grenoble, France. ${ }^{7}$ Instituto de Astronomía, Universidad Nacional Autónoma de México, Mexico City, Mexico. ${ }^{8}$ Unidad Mixta Internacional Franco-Chilena de Astronomía (CNRS UMI 3386), Departamento de Astronomía, Universidad de Chile, Santiago, Chile. ${ }^{9}$. Physikalisches Institut, Universität zu Köln, Cologne, Germany. ${ }^{10}$ Faculdade de Engenharia, Universidade do Porto, Porto, Portugal. ${ }^{11}$ CENTRA, Centro de Astrofísica e Gravitação, Instituto Superior Técnico, Lisbon, Portugal. ${ }^{12}$ European Southern Observatory, Santiago, Chile. ${ }^{13}$ Max Planck Institute for Extraterrestrial Physics, Garching bei München, Germany. ${ }^{14}$ Sterrewacht Leiden, Leiden University, Leiden, The Netherlands. ${ }^{15}$ Faculdade de Ciências, Universidade de Lisboa, Lisbon, Portugal. ${ }^{16}$ LESIA, Observatoire de Paris, Université PSL, CNRS, Sorbonne Université, Université de Paris, Meudon, France. ${ }^{17}$ Max-Planck-Institute for Radio Astronomy, Bonn, Germany. ${ }^{18}$ Department of Physics, University of California, Berkeley, CA, USA. ${ }^{\bowtie}$ e-mail: rebeca.garcialopez@ucd.ie 


\section{Methods}

\section{Observations and data reduction}

TW Hya was observed with the VLTI instrument GRAVITY ${ }^{30}$ on 21 January 2019 using the four 8-m Unit Telescopes of the European Southern Observatory (see Extended Data Table 1). The target was observed in single-field combined polarization mode (that is, fringes were tracked and servoed on the target itself), using the MACAO on-axis adaptive optics system. The data on the fringe tracker detector were recorded at low spectral resolution $(R \approx 23)$ with a detector integration time per interferogram of $0.85 \mathrm{~ms}$, whereas the science detector was working at high spectral resolution $\left(R \approx 4,000\right.$, that is, $\left.\Delta V \approx 70 \mathrm{~km} \mathrm{~s}^{-1}\right)$ and with a detector integration time per interferogram of $30 \mathrm{~s}$.

The data were reduced using the GRAVITY pipeline version 1.3.0 (ref. ${ }^{31}$ ). The atmospheric transfer function was calibrated using the calibrators HD 91937 and HD 95470 (see Extended Data Table 1). The spectrum of TW Hya was obtained by averaging the high-spectral-resolution spectra recorded in the four photometric channels. Standard telluric correction was also applied to the spectrum using HD 95470 (SpT K2/3 III) as a telluric standard star. Finally, the spectrum was flux-calibrated assuming a Two Micron All-Sky Survey (2MASS) K-band magnitude of 7.3 for TW Hya. The wavelength calibration of the spectra was refined using several telluric absorption lines present in the spectrum. An average shift of about $4 \AA$ was applied to the data.

\section{Interferometric observables}

VLTI-GRAVITY observations of TW Hya provided us with the K-band spectrum of the source, six spectrally dispersed visibilities (which give a measure of the size of the object, with $V=1$ indicating a point source and $V=0$ indicating a fully resolved object) and differential phases (which measure the photocentre shift of the line with respect to the continuum) and four closure phases (which provide a measure of the asymmetry of the continuum and/or line emission) (see Fig. 1).

The spectrum of TW Hya shows bright Bry $2.166 \mu \mathrm{m}$ line emission, along with Na I $2.206 \mu \mathrm{m}$, and $\mathrm{Na}$ I $2.209 \mu \mathrm{m}$, and rovibrational CO in absorption. No interferometric signal is detected for any of these lines except the Bry line. A small differential phase signature of $2^{\circ}$ in the $\mathrm{Br} \gamma$ line is detected along the two longest baselines. No closure phases were detected within the errors.

The continuum visibilities point towards a very compact circumstellar environment around TW Hya with measured continuum visibilities above about 0.95 in all our baselines. Interestingly, the total visibilities within the line decreases with respect to the continuum visibilities, indicating that the sum of the Bry emitting region plus the continuum contribution (including the stellar plus circumstellar environment) is more extended than the continuum alone. However, it should be noted that the total visibility is not just the sum of each visibility component but is weighted by the flux of each component. In other words, assuming that the level of the continuum within and outside the line is the same and that the differential phase is zero, then: $V_{\text {tot }} F_{\text {tot }}=V_{\text {cont }} F_{\text {cont }}+V_{\text {line }} F_{\text {line, }}$, with $V_{\text {cont }} F_{\text {cont }}=V_{*} F_{*}+V_{\text {circ }} F_{\text {circ }}$; and $F_{\text {tot }}=F_{\text {cont }}+F_{\text {line. }}$ In these expressions, $F_{\text {tot }}, F_{\text {cont }}, F_{\text {line, }}$ and $V_{\text {tot }}, V_{\text {cont }}, V_{\text {line }}$ are the total, continuum and line fluxes and visibilities, respectively; and $F_{*}, F_{\text {circ }}$, and $V_{*}, V_{\text {circ }}$ are the stellar and circumstellar continuum fluxes and visibilities. To further investigate the circumstellar and Bry line emitting region the contribution from the star and the overall continuum emission must be removed from the measured visibilities.

\section{Circumstellar continuum emitting region}

As mentioned above, to estimate the size of the continuum circumstellar emitting region the emission from the star must be removed. In doing so, a K-band to stellar flux ratio of 1.18 was assumed ${ }^{14}$. As we expected to be able to marginally resolve TW Hya owing to its close distance (that is, $V_{*}<1$ on our longest baselines), we took the conservative approach of fitting two Gaussian components to the continuum visibilities measured by GRAVITY, one corresponding to the central star plus an additional component due to the circumstellar disk (assumed to be inclined, that is, the inclination $(i)$ and position angle (PA) were allowed to be free parameters). Using this approach, our best-fitting model corresponds to two Gaussians with full-width-at-half-maximum values of $\mathrm{FWHM}_{*}=0.20 \pm 0.03$ mas and $\mathrm{FWHM}_{\text {circ }}=1.30 \pm 0.04$ mas, respectively. The $i$ and PA values of the latter component are consistent with a nearly face-on structure as reported by $\mathrm{ALMA}^{11}$. However, owing to the lack of long baselines, along with the nearly face-on geometry, we cannot provide stringent constraints on the $i$ and PA values of the system, and from now on we will assume ALMA measurements of $i \approx 5^{\circ}$ and $\mathrm{PA} \approx 32^{\circ}$ as our fiducial values. Coming back to the size of the emitting region, the derived FWHM values correspond to a stellar radius of $R_{*}=(1.29 \pm 0.19) R_{\odot}$ and $R_{\text {circ }}=(8.39 \pm 0.21) R_{\odot}$ assuming a distance of $60 \mathrm{pc}\left(\right.$ ref. $\left.{ }^{41}\right)$. The retrieved stellar and circumstellar radii are in good agreement with previous values found in the literature ${ }^{14,20,32,33}$. If a lower value of the observed $\mathrm{K}$-band to stellar flux ratio of 1.10 is assumed ${ }^{34,35}$, it would provide a worse fit, with stellar and circumstellar radii with much larger errors, namely, $R_{*}=0.05 \pm 0.11$ mas (that is, $(0.68 \pm 1.42) R_{\odot}$ ), and $R_{\text {circ }}=0.70 \pm 0.13$ mas (that is, $(9.03 \pm 1.68) R_{\odot}$ ).

\section{Continuum-subtracted Bry line visibilities}

The size of the Bry line emitting region can be estimated by assuming that the total visibilities within the Bry line are due to the contribution of the line emitting region plus the continuum component. In this way, the pure (or continuum compensated) Bry line visibilities can be derived by subtracting the continuum contribution from the total line visibilities following ${ }^{36}$ :

$$
V_{\text {cont }} V_{\text {tot }} \mathrm{e}^{\mathrm{i} \phi^{\prime}}=\frac{V_{\text {cont }}}{F_{\text {cont }}+F_{\text {line }}}\left(F_{\text {cont }} V_{\text {cont }}+F_{\text {line }} V_{\text {line }} \mathrm{e}^{\mathrm{i} \Delta \phi}\right)
$$

where $\phi^{\prime}$ is the differential phase in the line, and $\Delta \Phi$ is the difference of the Fourier phases of the continuum and line components, that is $\Delta \Phi\left(B / \lambda_{\text {line }}\right)=\Phi_{\text {cont }}\left(B / \lambda_{\text {line }}\right)-\Phi_{\text {line }}\left(B / \lambda_{\text {line }}\right)$. Thus the errors on the continuum compensated visibilities have been estimated taking into account the error on the continuum and total visibilities (assuming the root-mean-square value as a conservative error), and the differential phase errors.

Initially, the continuum-compensated Bry line visibilities were computed at three velocity channels at radial velocities of approximately $-33 \mathrm{~km} \mathrm{~s}^{-1}, 4 \mathrm{~km} \mathrm{~s}^{-1}$ and $40 \mathrm{~km} \mathrm{~s}^{-1}$, and with a line-to-continuum ratio higher than $10 \%$. For all six baselines, the continuum-compensated $\mathrm{Br} \gamma$ line visibilities measured at each spectral channel are roughly the same within the errors. Therefore, the weighted mean of the three pure line visibilities for each baseline was computed and used to derive the size of the Bry line emitting region. The average pure Bry line visibilities are shown in Figs. 1, 2. The Bry line emitting region is marginally resolved only for the longest projected baselines (more than about $60 \mathrm{~m}$ ), meaning that the emitting region is very compact.

To derive the size of the Bry line emitting region, we computed a geometric model of the Bry line continuum-compensated visibilities using a Gaussian fit. As for the continuum, we fixed the $i$ and PA to the values derived by ALMA and we fitted the line visibilities with only the Gaussian FWHM as a free parameter. The best-fit result is shown in Table 1, and it corresponds to a radius of the $\mathrm{Br} \gamma$ line emitting region of $R_{\mathrm{Br \gamma}}=0.35 \pm 0.02$ mas or $R_{\mathrm{Br \gamma}}=(4.5 \pm 0.26) R_{\odot}$, assuming a distance of about 60 pc to TW Hya (Fig. 2 and Extended Data Fig. 1 ).

To probe the effect of the assumed $F_{\text {tot }} / F_{\text {cont }}$ flux ratio on our results, we have repeated the analysis, varying this ratio by $10 \%$. The results $\left(R_{\mathrm{Br \gamma}}^{-10 \%}=0.37 \pm 0.05\right.$ mas; $R_{\mathrm{Br \gamma}}^{-10 \%}=0.33 \pm 0.02$ mas $)$ are consistent with the previous one within the error bars.

\section{Continuum-subtracted Bry line differential phase}

As for the case of the visibilities, the contribution of the continuum to the differential phase can be removed. This type of analysis is especially 


\section{Article}

useful when the measured photocentre shift of the line is weak ${ }^{37,38}$. Following ref. ${ }^{36}$, the displacement of the photocentre of the line at any given wavelength can be derived from:

$$
\sin (\Delta \phi)=\sin (\phi) \times \frac{\left|F_{\text {tot }} V_{\text {tot }}\right|}{\left|F_{\text {line }} V_{\text {line }}\right|}
$$

The displacement of the photocentre of the emission at any given wavelength $\delta$ is then:

$$
\delta=-\Delta \phi \frac{\lambda}{2 \pi B}
$$

where $B$ is the length of the baseline. The upper limit of the differential phase is about $1^{\circ}$. This translates into a maximum value of $\Delta \phi_{\max }^{\mathrm{Br \gamma}}=-6.2^{\circ}$, equivalent to a maximum displacement of $\delta_{\max } \approx 3.8 R_{\odot} \approx 4.9 R_{*}$. This value is very similar to the one derived from the continuum-subtracted visibilities.

\section{Rim radius}

We can estimate the rim radius (or the distance from the star where silicates sublimates) using equation (11) of Dullemond et al. ${ }^{39}$ under the assumption that the pressure scale height is a small fraction of $R$. For the temperature $(4,000 \mathrm{~K})$ and radius $\left(R \approx 1.2 R_{\odot}\right)$ assumed here for TW Hya, a rim radius of $R_{\text {rim }} \approx 7.5 R_{*}$ is found for a sublimation temperature of $1,500 \mathrm{~K}$.

\section{Corotation radius}

The corotation radius depends on the stellar mass, radius and rotation velocity. This latter is uncertain owing to the low inclination of TW Hya with respect to the line of sight. Estimates of the rotation velocity ranges from $80 \pm 34 \mathrm{~km} \mathrm{~s}^{-1}$ (assuming $v \sin i=7 \pm 3 \mathrm{~km} \mathrm{~s}^{-1}\left(\right.$ ref. $^{15}$ ) and a disk inclination of $5^{\circ}$; ref. ${ }^{11}$ ) to about $17.4 \mathrm{~km} \mathrm{~s}^{-1}$ (assuming a rotation period of $P=3.56$ days; ref. ${ }^{40}$ ). Taking these values, and a stellar mass and radius of $0.58 \mathrm{M}_{\odot}$ and $1.22 R_{\odot}$ (ref. ${ }^{15}$ ), we find a corotation radius of $R_{\mathrm{co}} \approx 6.5 R_{*}-7.1 R_{*}$. The corotation radius is noticeably larger than $R_{\mathrm{Br \gamma}}$, supporting our hypothesis that the Bry size measures the radius of the magnetosphere, probably tracing the width of the region containing accretion columns.

\section{Data availability}

This work is based on observations collected at the European Southern Observatory (ESO) under ESO programme 0102.C-0408(C). The raw data are publicly available in the ESO Science Archive Facility.
The reduced data that support the findings of this study are available from the corresponding author under reasonable request.

30. Gravity Collaboration. First light for GRAVITY: phase referencing optical interferometry for the Very Large Telescope Interferometer. Astron. Astrophys. 602, A94 (2017).

31. Lapeyrere, V. et al. GRAVITY data reduction software. In Proc. SPIE Optical and Infrared Interferometry IV Vol. 9146, 91462D (SPIE, 2014).

32. Eisner, J. A., Chiang, E. I. \& Hillenbrand, L. A. Spatially resolving the inner disk of TW Hydrae. Astrophys. J. 637, L133-L136 (2006).

33. Sokal, K. R. et al. Characterizing TW Hydra. Astrophys. J. 853, 120 (2018).

34. Eisner, J. A. et al. Time-variable accretion in the TW Hya star/disk system. Astrophys. J. 722, L28-L32 (2010)

35. Vacca, W. D. \& Sandell, G. Near-infrared spectroscopy of TW Hya: a revised spectral type and comparison with magnetospheric accretion models. Astrophys. J. 732, 8 (2011).

36. Weigelt, G. et al. Near-infrared interferometry of $\eta$ Carinae with spectral resolutions of 1500 and 12000 using AMBER/VLTI. Astron. Astrophys. 464, 87-106 (2007).

37. Whelan, E. T. et al. Classical T Tauri-like outflow activity in the brown dwarf mass regime. Astrophys. J. 706, 1054-1068 (2009).

38. Agra-Amboage, $V$. et al. Origin of the wide-angle hot $\mathrm{H}_{2}$ in DG Tauri. New insight from SINFONI spectro-imaging. Astron. Astrophys. 564, A11 (2014).

39. Dullemond, C. P., Dominik, C. \& Natta, A. Passive irradiated circumstellar disks with an inner hole. Astrophys. J. 560, 957-969 (2001).

40. Setiawan, J. et al. A young massive planet in a star-disk system. Nature $\mathbf{4 5 1}, \mathbf{3 8 - 4}$ (2008)

41. Bailer-Jones, C. A. L., Rybizki, J., Fouesneau, M., Mantelet, G. \& Andrae, R. Estimating distance from parallaxes. IV. Distances to 1.33 billion stars in Gaia Data Release 2. Astron. J. 156, 58 (2018)

Acknowledgements We thank C. Manara for providing the XSHOOTER spectrum of TW Hya and the template of the stellar photosphere. This material is based upon works supported by Science Foundation Ireland under grant number 18/SIRG/5597. M.K. was funded by the Irish Research Council (IRC), grant GOIPG/2016/769. R.F. acknowledges support from the Chalmers Initiative on Cosmic Origins ( $\mathrm{ClCO}$ ) postdoctoral fellowship. A.C.G. and T.P.R. have received funding from the European Research Council (ERC) under the European Union's Horizon 2020 research and innovation programme (grant agreement number 743029). A.N. acknowledges the hospitality of DIAS. A.A., M.F. and P.J.V.G. acknowledge funding by the Fundação para a Ciência e a Tecnologia, with grant references UID/FIS/00099/2013 and SFRH/ BSAB/142940/2018. Th.H. acknowledges support from the European Research Council unde the Horizon 2020 Framework Program via the ERC Advanced Grant Origins 832428

Author contributions GRAVITY is developed as a collaboration by the Max Planck Institute for Extraterrestrial Physics, LESIA of Paris Observatory and the IPAG of Université Grenoble Alpes/ CNRS, the Max Planck Institute for Astronomy, the University of Cologne, the Centro Multidisciplinar de Astrofisica Lisbon and Porto, and the European Southern Observatory. Authors from these institutes contributed to the concept, design, assembly, instrumental tests, science cases, verification and implementation of GRAVITY and its subsystems, and the data reduction pipeline. P.J.V.G. conducted the observations. R.G.L., K.P. and M.K. reduced the data R.G.L. and M.K. analysed the data. A.N. estimated the location of the disk rim, and the corotation and truncation radii. R.F. performed the model fitting of the continuum-subtracted visibilities. R.G.L. wrote the manuscript. A.N., T.P. and A.C.G. edited the manuscript. R.G.L., A.N.,

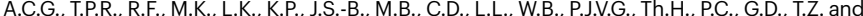
R.G. discussed the results and their implications, and commented on the manuscript.

Competing interests The authors declare no competing interests.

Additional information

Correspondence and requests for materials should be addressed to R.G. Reprints and permissions information is available at http://www.nature.com/reprints. 


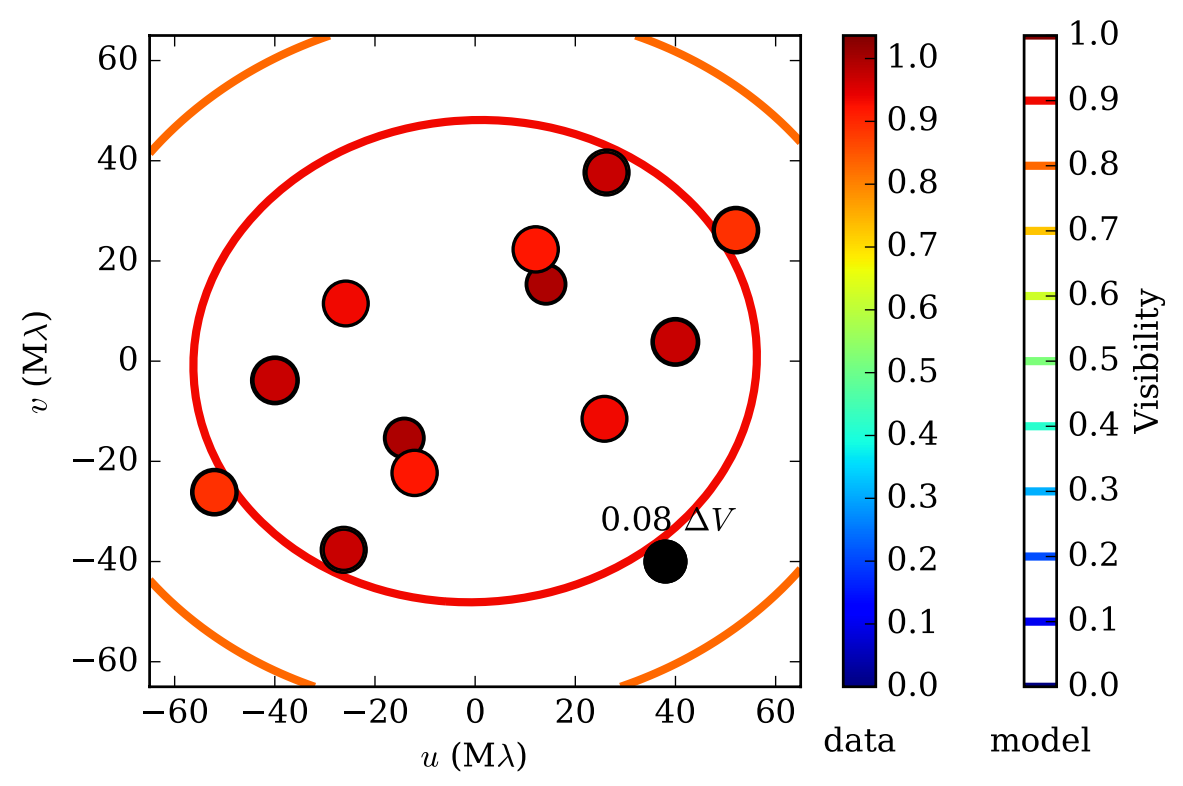

Extended Data Fig. 1 | Best-fit model to the continuum-subtracted Bry line visibilities. Continuum subtracted visibilities are represented in colour in the $u-v$ plane. The symbol size indicate the error of each single data point.
For comparison, the average visibility error is represented by the dark full circle at the bottom right of the figure. Contours represent the visibility values of the best two-dimensional Gaussian model. 


\section{Article}

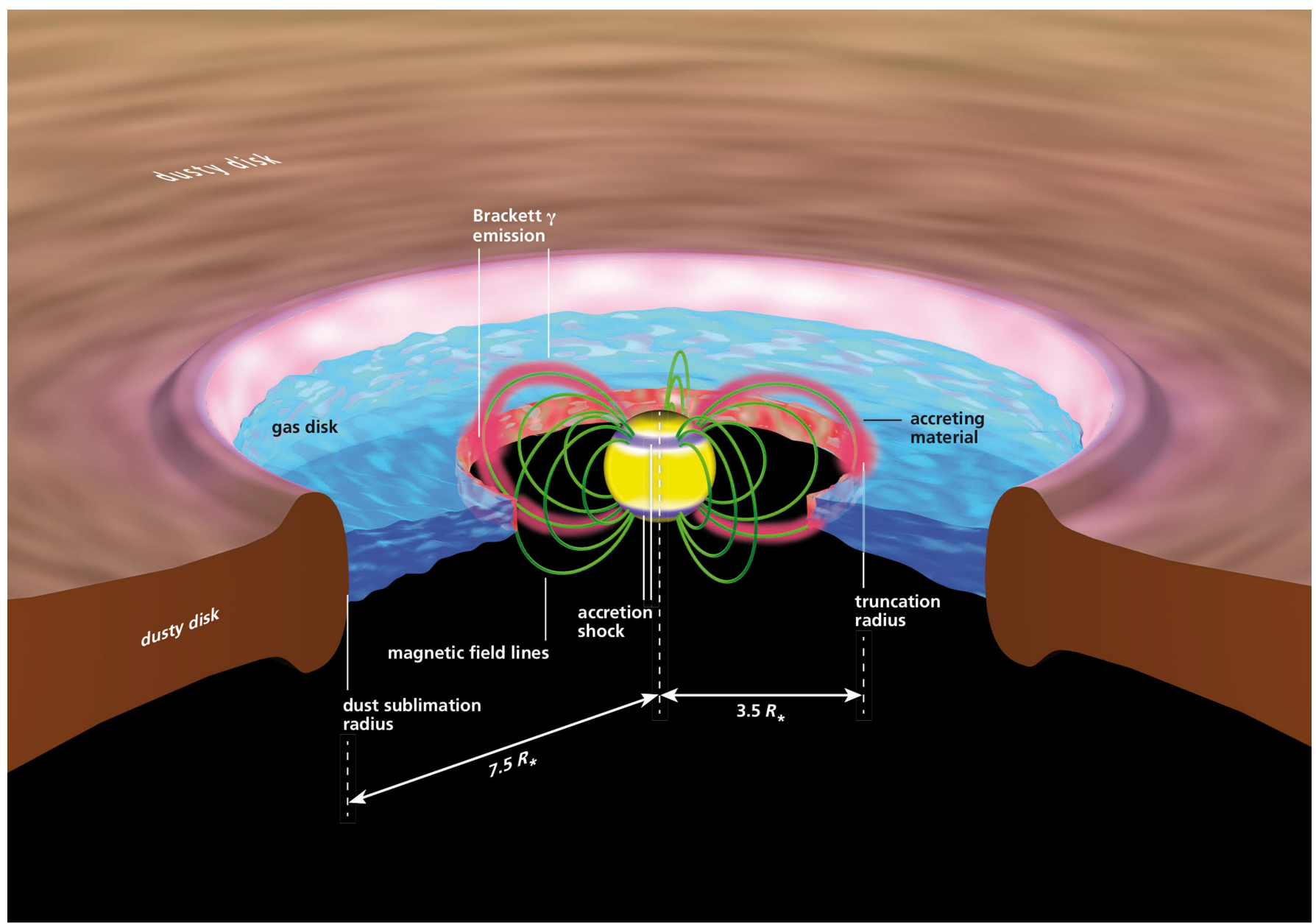

Extended Data Fig. 2 |Sketch of the inner-disk region of TW Hya. The main features of the inner disk are represented: the dusty disk (brown), the dust sublimation radius located at about 7.5R*, the inner gaseous disk (blue), truncated by the stellar magnetosphere (red) at about $3.5 R_{*}$, along with the $\mathrm{Br} \gamma$ line emitting region, which is probably tracing the width of the accretion columns. 
Extended Data Table 1 | Observation log of the VLTI GRAVITY+UT high-resolution observations of TW Hya

\begin{tabular}{cccccc|cc}
\hline UT Date & Tot. Int. & DIT $^{*}$ & NDIT $\dagger$ & Proj. baselines & PA $\ddagger$ & Calibrators & UD diameter \\
& {$[\mathrm{s}]$} & {$[\mathrm{s}]$} & {$[\mathrm{s}]$} & {$[\mathrm{m}]$} & {$[\circ]$} & & {$[\mathrm{mas}]$} \\
\hline $2019-01-21$ & 3900 & 30 & 10 & $45,54,61$, & $42,28,114$, & HD 91937, & $0.279 \pm 0.004$, \\
& & & & $86,99,126$ & $84,34,63$ & HD 95470 & $0.367 \pm 0.005$ \\
\hline
\end{tabular}

UT, Unit Telescope; Tot. Int., total integration; Proj., projected.

*DIT, detector integration time per interferogram.

†NDIT, number of interferograms.

¥PA, baseline position angle from the shortest to longest baseline.

§UD diameter, the calibrator uniform-disk diameter (K band) was taken from the SearchCal tool available at http://www.jmmc.fr/searchcal. 